\title{
VEGF-B: a thing of beauty
}

\author{
Xuri $\mathrm{Li}^{1}$ \\ ${ }^{1}$ National Institutes of Health/National Eye Institute, Rockville, 20852, Maryland, USA \\ Cell Research (2010) 20:741-744. doi:10.1038/cr.2010.77; published online 8 June 2010
}

More than a decade ago, when we first embarked on our journey to delineate the biological function of vascular endothelial growth factor B (VEGF-B), we had a hard time comprehending why VEGF-B was needed. In mice, genetic deletion of VEGF-B seemed to be harmless, since the VEGF-B null mice, to a large extent, can still live a fairly normal life [1]. Moreover, overexpression of VEGF-B in different mouse tissues, such as the skin or skeletal muscle, did not seem to result in any obvious phenotype [2]. Due to these seemingly cometo-nothing findings, many researchers lost their scientific interests in VEGF-B. However, new discoveries on VEGF-B function have recently begun to surprise us - the latest one being that VEGF-B plays an important role in modulating fat utilization. Deeper scrutiny demonstrated that VEGF-B deficient mice display greater amount of body fat and weight due to impaired fatty acid (FA) uptake by the endothelium, as shown elegantly in a recent study from Dr Ulf Eriksson's laboratory [3].

VEGF-B was discovered in 1996 as a VEGF homologue [4]. VEGF-B is produced as a secreted homodimer. Due to alternative splicing, the $V E G F-B$ gene gives rise to two isoforms, VEGF-B ${ }_{167}$ and VEGF- $\mathrm{B}_{186}$, which are homodimers of about 42 and $60 \mathrm{kDa}$, respectively. VEGF-B $_{186}$ can be proteolytically processed at Arg127 and give rise to a 34

Correspondence: Xuri Li

E-mail: lixur@nei.nih.gov
kDa dimer. VEGF-B ${ }_{167}$ has a heparinbinding domain, so that upon secretion, VEGF-B ${ }_{167}$ binds to cell-surface heparan sulphate proteoglycans. By contrast, VEGF-B ${ }_{186}$ does not contain the heparin-binding domain and therefore is more soluble. VEGF-B binds to vascular endothelial growth factor receptor-1 (VEGFR-1) and neuropilin-1 (NRP-1) [5, 6]. VEGF-B is expressed early during fetal development in mice, and remains abundantly expressed in most tissues and organs in adult mice, especially in the cardiac myocytes, skeletal muscles and neuronal tissues [7]. $V E G F-B_{167}$ is the predominant isoform expressed in most tissues and organs, accounting for more than $80 \%$ of the total $V E G F-B$ transcripts, while $V E G F-B_{186}$ is expressed at lower levels and in a limited number of tissues [7]. For many years, research efforts on VEGF-B have focused on its speculated angiogenic activities, based on its high sequence homology and similar receptor binding pattern to VEGF, a prototype angiogenic factor. However, studies along this line, most of the time, led to inconsistent results [8]. Compared with the other VEGF family members, VEGF-B has received much less attention thus far.

Recent years have witnessed several advances in VEGF-B biology. First, different groups have shown that VEGF-B is a potent neuroprotective factor [8-10]. Second, it is recently recognized that VEGF-B has an ischemic myocardiumspecific angiogenic activity while being minimally angiogenic in most of the other organs $[2,11,12]$. Compared with these findings, the more recent discovery by Dr Eriksson' group, perhaps is more unexpected and striking. In this study, Hagberg et al. [3] showed that VEGF-B is a critical regulator of energy metabolism by regulating fatty acid uptake.

In their recent study, Hagberg et al. [3] provided several lines of evidence at different levels to show that VEGF-B has a unique and critical role in regulating fatty acid transportation. First, the authors conducted bioinformatic analysis of published microarray data and found that VEGF-B expression was closely associated with the expression of nuclear-encoded mitochondrial genes under different conditions in mice. This association appears to be specific to VEGF-B, since the other VEGF family members, such as VEGF and PlGF, do not display the same type of association in their expression. These observations at gene expression level thus pointed to a potential role of VEGF-B in energy metabolism. The authors then went on and verified the significance of the above observations using cultured cells, and found that, in endothelial cells, VEGF-B stimulation upregulated the expression of the fatty acid transport proteins (FATPs), which are a family of proteins needed for fatty acid transportation across the endothelium. Indeed, in a two-liquid-compartment endothelial cell culture assay, VEGF-B treatment increased trans-endothelial transfer of ${ }^{14} \mathrm{C}$-labelled oleic acid from the upper to the basal liquid compartment, 
A

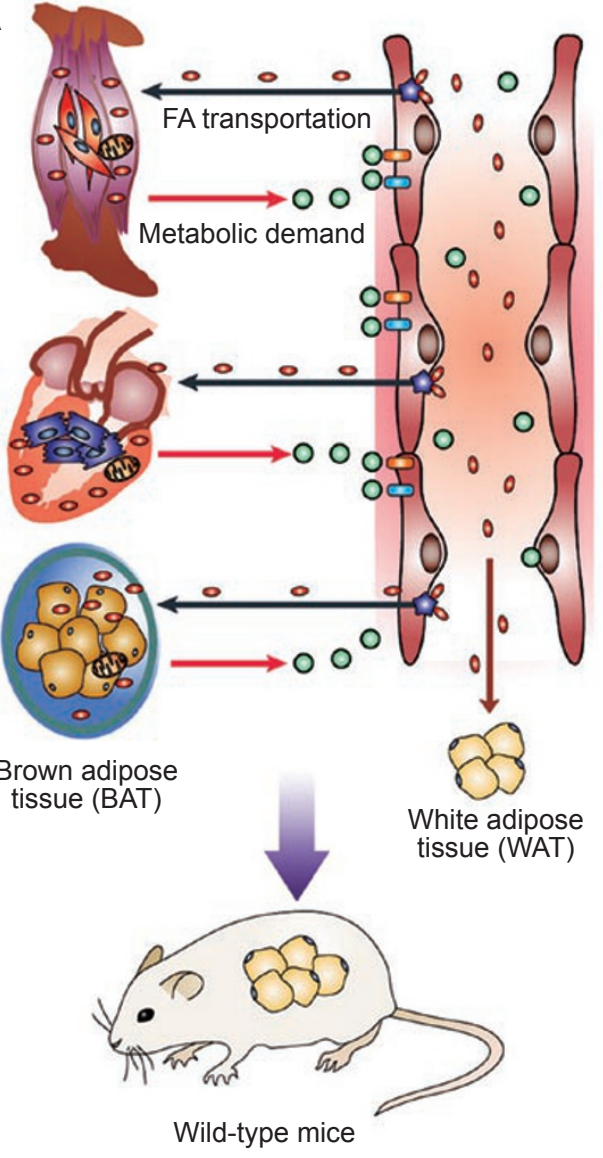

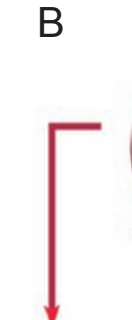

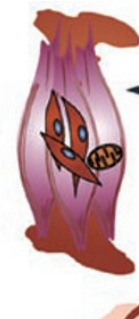

Apoptosis

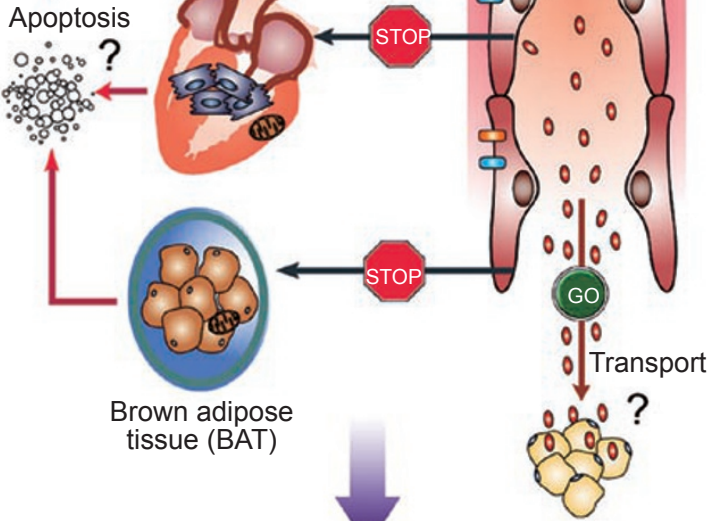

White adipose tissue (WAT)
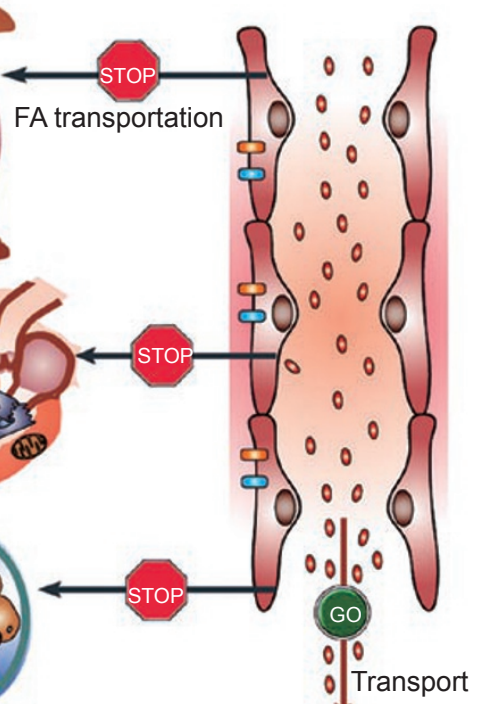

\section{(n)}

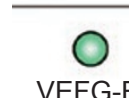

VEFG-B<smiles>C=C=C</smiles>

Endothelial cell
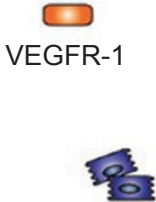

Cardiomyocyte

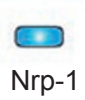

Nrp-1 
and resulted in increased amount of body fat and weight in the VEGF-B null mice (Figure 1). Furthermore, as a compensation for the reduced lipid utilization, the VEGF-B deficient mice had an increased glucose uptake and utilization in their hearts as an alternative source of energy. Mechanistically, the authors revealed that the effect of VEGF-B on endothelial FA uptake was mediated by Flt-1 and Nrp-1. This notion is supported by the findings that in mice lacking functional Flt-1 and Nrp1, the cardiac expression of FATPs was decreased. Indeed, the authors also found that the Nrp1 deficient mice displayed a similar defect in fatty acid uptake to peripheral tissues.

One common exciting aspect of all breakthroughs is that they always lead to interesting questions.

There appears to be an apparent tissue specificity of VEGF-B action in affecting fatty acid metabolism, since VEGF-B deficient mice display an impaired FA uptake in their BATs, but an increased FA uptake in their WATs. Why does VEGF-B deficiency not blunt FA uptake in the WATs, since VEGF-B is a secreted protein and can be found in the blood stream in different tissues? Are Flt-1 and Nrp-1 not expressed by the endothelium in WAT? Or, does FA uptake in WATs utilize different molecules other than VEGF-B? Furthermore, it is known that Flt-1 and Nrp-1 are expressed by many cell types. Does VEGF-B affect FA uptake in other types of cells apart from vascular endothelial cells?

It would be interesting to know the direct or indirect biological consequences of the impaired FA uptake in the VEGF-B deficient heart, muscle and BAT. VEGF-B has been shown to be a potent survival factor [8, 9]. VEGF-B treatment increased the survival of different cell types, including neurons $[9,10]$, blood vessels [11], and cardiac myocytes $[13,14]$. It would be interesting to see whether the survival effect of VEGF-B is linked to its FA-transport function, or, whether they are two separate pathways. It is noteworthy that docosahexaenoic acid (DHA), one major n-3 fatty acid, is specifically required for retinal neuronal survival [15]. This has indicated a link between FA uptake and neuronal survival, in both of which VEGF-B plays an important role.

Also, it remains unclear why VEGF$\mathrm{B}_{186}$, the more diffusible form of VEGF$\mathrm{B}$, was more effective than the heparinbinding form of VEGF-B, VEGF-B ${ }_{167}$, in inducing FATP expression. In the study by Hagberg et al., the co-expression of VEGF-B with the mitochondrial protein genes was the initial indication of a role of VEGF-B in energy metabolism. However, VEGF- ${ }_{186}$ is secreted and soluble and can be transported freely to different tissues through the blood stream. Instead, one would hypothesize that VEGF-B ${ }_{167}$, which is heparin binding and less soluble with a greater tissue specificity, might be more likely to fulfill the tissue-specific demand of FA uptake to match up with the oxidative capacity of that specific tissue.

It is particularly interesting to note that VEGF-B deficiency results in greater body fat mass and weight in mice. Given that obesity is becoming an epidemic in developed and developing countries and causes significant morbidity and mortality, it will be interesting to further delve into the expression and functional status of VEGF-B in obese patients, and to verify whether obesity in human may potentially be associated with any functional defect of VEGF-B, and if so, whether fortified VEGF-B expression could help fight against obesity. Moreover, it is known that fatty acid uptake affects numerous biological processes in the body, including cardiovascular, neurological and immune functions. It is therefore reasonable to expect that the discovery of a new critical player in lipid uptake, such as VEGF-B, might open up new therapeutic possibilities to tackle pathological lipid accumulation in obesity, diabetes, cardiovascular and other diseases.
We await the next surprise from VEGF-B.

\section{Acknowledgments}

The author thanks Dr Fan Zhang (National Institutes of Health/National Eye Institute) for the artistic work for the figure, and thanks Dr Lijin Dong (National Institutes of Health/National Eye Institute) and the laboratory members for the helpful comments.

\section{References}

1 Aase K, von Euler G, Li X, et al. Vascular endothelial growth factor-B-deficient mice display an atrial conduction defect. Circulation 2001; 104:358-364.

2 Li X, Tjwa M, Van Hove I, et al. Reevaluation of the role of VEGF-B suggests a restricted role in the revascularization of the ischemic myocardium. Arterioscler Thromb Vasc Biol 2008; 28:1614-1620.

3 Hagberg CE, Falkevall A, Wang X, et al. Vascular endothelial growth factor B controls endothelial fatty acid uptake. Nature 2010; 464:917-921.

4 Olofsson B, Pajusola K, Kaipainen $\mathrm{A}$, et al. Vascular endothelial growth factor B, a novel growth factor for endothelial cells. Proc Natl Acad Sci USA 1996; 93:2576-2581.

5 Olofsson B, Korpelainen E, Pepper MS, et al. Vascular endothelial growth factor B (VEGF-B) binds to VEGF receptor-1 and regulates plasminogen activator activity in endothelial cells. Proc Natl Acad Sci USA 1998; 95:11709-11714.

6 Makinen T, Olofsson B, Karpanen T, et al. Differential binding of vascular endothelial growth factor B splice and proteolytic isoforms to neuropilin-1. $J$ Biol Chem 1999; 274:21217-21222.

7 Li X, Aase K, Li H, von Euler G, Eriksson U. Isoform-specific expression of VEGF-B in normal tissues and tumors. Growth factors 2001; 19:49-59.

8 Li X, Lee C, Tang Z, et al. VEGF-B: A survival, or an angiogenic factor? Cell Adh Migr 2009; 3:322-327.

9 Li Y, Zhang F, Nagai N, et al. VEGF-B inhibits apoptosis via VEGFR-1-mediated suppression of the expression of BH3-only protein genes in mice and rats. J Clin Invest 2008; 118:913-923.

10 Poesen K, Lambrechts D, Van Damme 
$\mathrm{P}$, et al. Novel role for vascular endothelial growth factor (VEGF) receptor-1 and its Ligand VEGF-B in motor neuron degeneration. J Neurosci 2008; 28:10451-10459.

11 Zhang F, Tang Z, Hou X, et al. VEGF-B is dispensable for blood vessel growth but critical for their survival, and VEGF-B targeting inhibits pathological angiogenesis. Proc Natl Acad Sci USA 2009; 106:6152-6157.

12 Lahteenvuo JE, Lahteenvuo MT, Kive- la A, et al. Vascular endothelial growth factor-B induces myocardium-specific angiogenesis and arteriogenesis via vascular endothelial growth factor receptor-1- and neuropilin receptor-1-dependent mechanisms. Circulation 2009; 119:845-856.

13 Karpanen T, Bry M, Ollila HM, et al. Overexpression of vascular endothelial growth factor-B in mouse heart alters cardiac lipid metabolism and induces myocardial hypertrophy. Circ Res
2008; 103:1018-1026.

14 Tirziu D, Chorianopoulos E, Moodie $\mathrm{KL}$, et al. Myocardial hypertrophy in the absence of external stimuli is induced by angiogenesis in mice. J Clin Invest 2007; 117:3188-3197.

15 Rotstein NP, Aveldano MI, Barrantes FJ, Politi LE. Docosahexaenoic acid is required for the survival of rat retinal photoreceptors in vitro. $J$ Neurochem 1996; 66:1851-1859. 